\title{
Bread and Stroke
}

\section{Fiorella Nawar, MD, Sylvia Orozco-Do, MD, Boutros El Haddad,MD}

\section{Introduction}

- Celiac disease is an autoimmune disease that can present with intestinal and extra-intestinal manifestations.

- Neurologic manifestations of Celiac disease such as ataxia, depression and stroke are rare.

- We describe a case of Celiac disease presenting as stroke.

\section{Case Presentation}

- A 48-year-old Caucasian male with negative history for hypertension, diabetes mellitus, hyperlipidemia, and smoking presented for the sudden onset of confusion and aphasia.

- An MRI of the brain showed the presence of acute ischemia of the left middle cerebral artery.

- Complete hypercoagulable state workup was within range of normal except for the presence of moderately elevated homocysteine at 21.4.

- Basic laboratory testing showed the presence of iron deficiency anemia with normal level of vitamin B12 and low normal folic acid level.

- Vitamin D level was low.

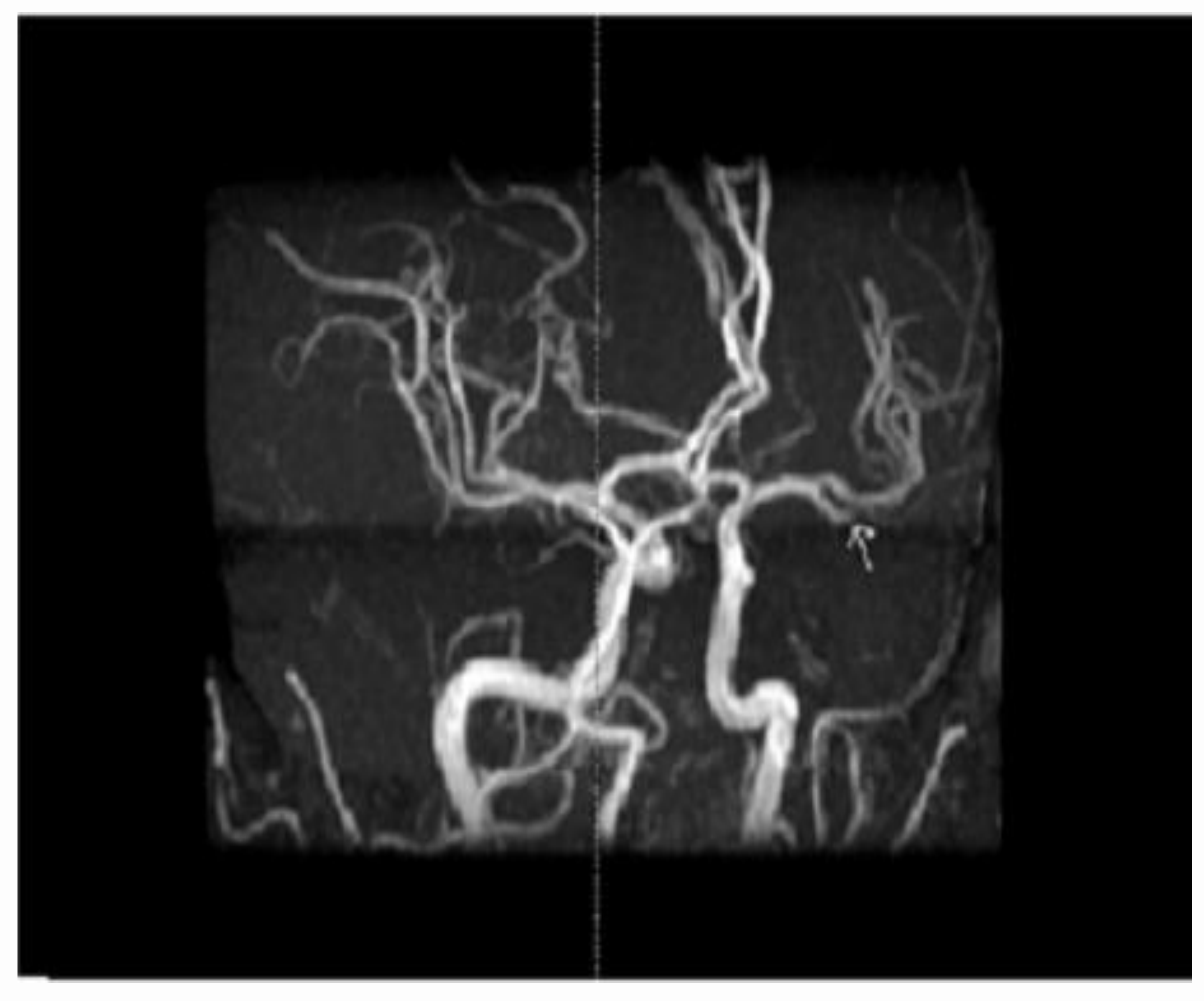

MRA showing L MCA occlusion

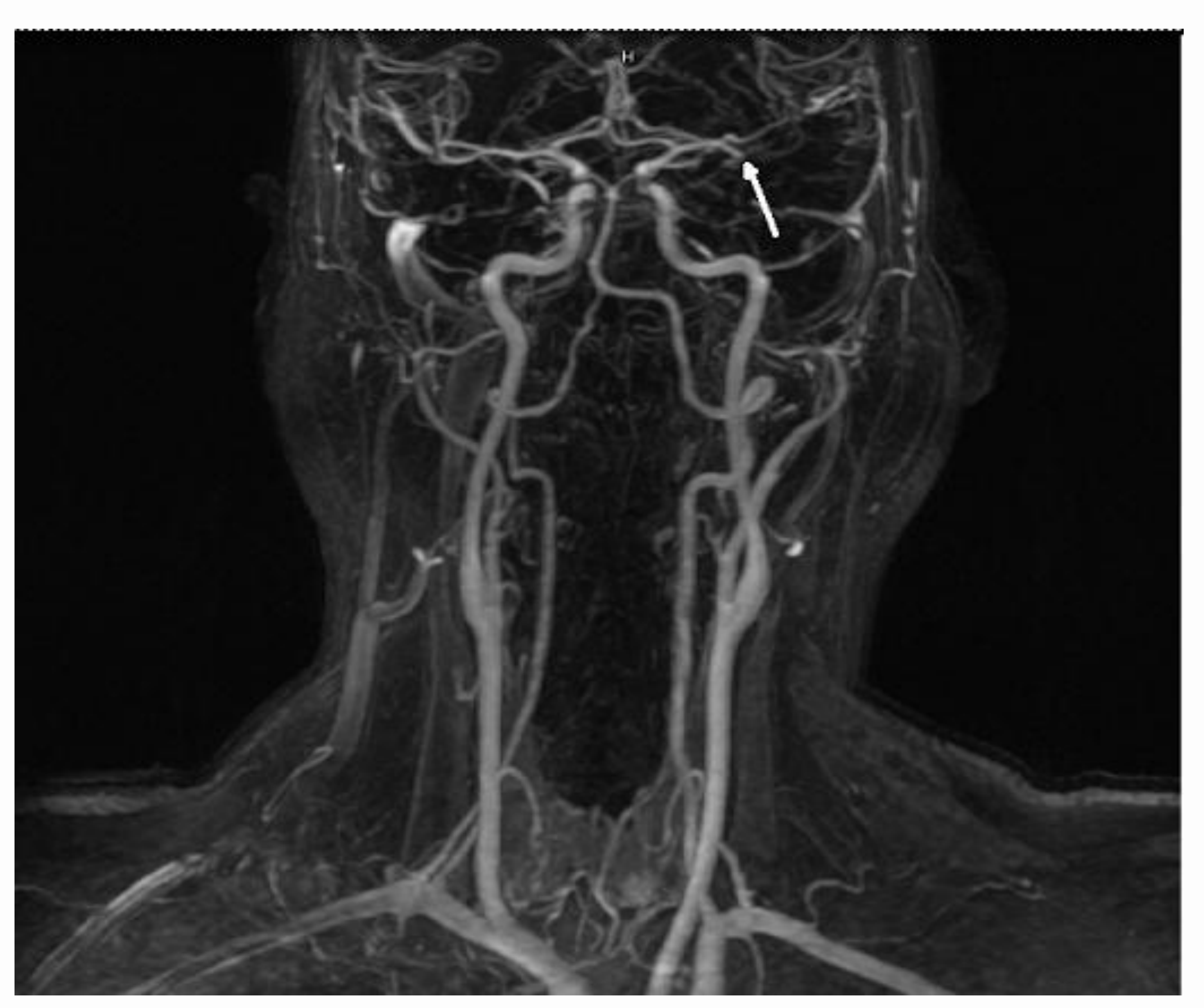

MRA coronal section showing L MCA occlusion

\section{References}

(1) Casella G, Bassotti G, Di Bella C, et al. Is hyperhomocysteinemia relevant in patients with celiac disease? World J Gastroenterol, 2011; 217(24):2941-2944.

(2) El Moutawakil B, Chourkani N, Sibai M, et al. Celiac disease and ischemic stroke. Revue Neurol (Paris) 2009; 165(11):962-966.
- Markers for celiac disease-antiendomysial and tissue transglutaminase antibodieswere positive.

- EGD with duodenal biopsy was not done in hospital.

- The patient was dismissed home on gluten free diet with suspected diagnosis of Celiac disease.

\section{Discussion}

- Celiac disease can have neurologic manifestation such as stroke.

- Hyperhomocysteinemia is observed in 20\% of newly diagnosed celiac disease.(1)

- Homocysteinemia is a risk factor for CHD and stroke.

- In patients with evidence of malabsorption and no obvious etiology for stroke, Celiac disease mediated by hyperhomocysteinemia should be considered a potential etiology.(2)

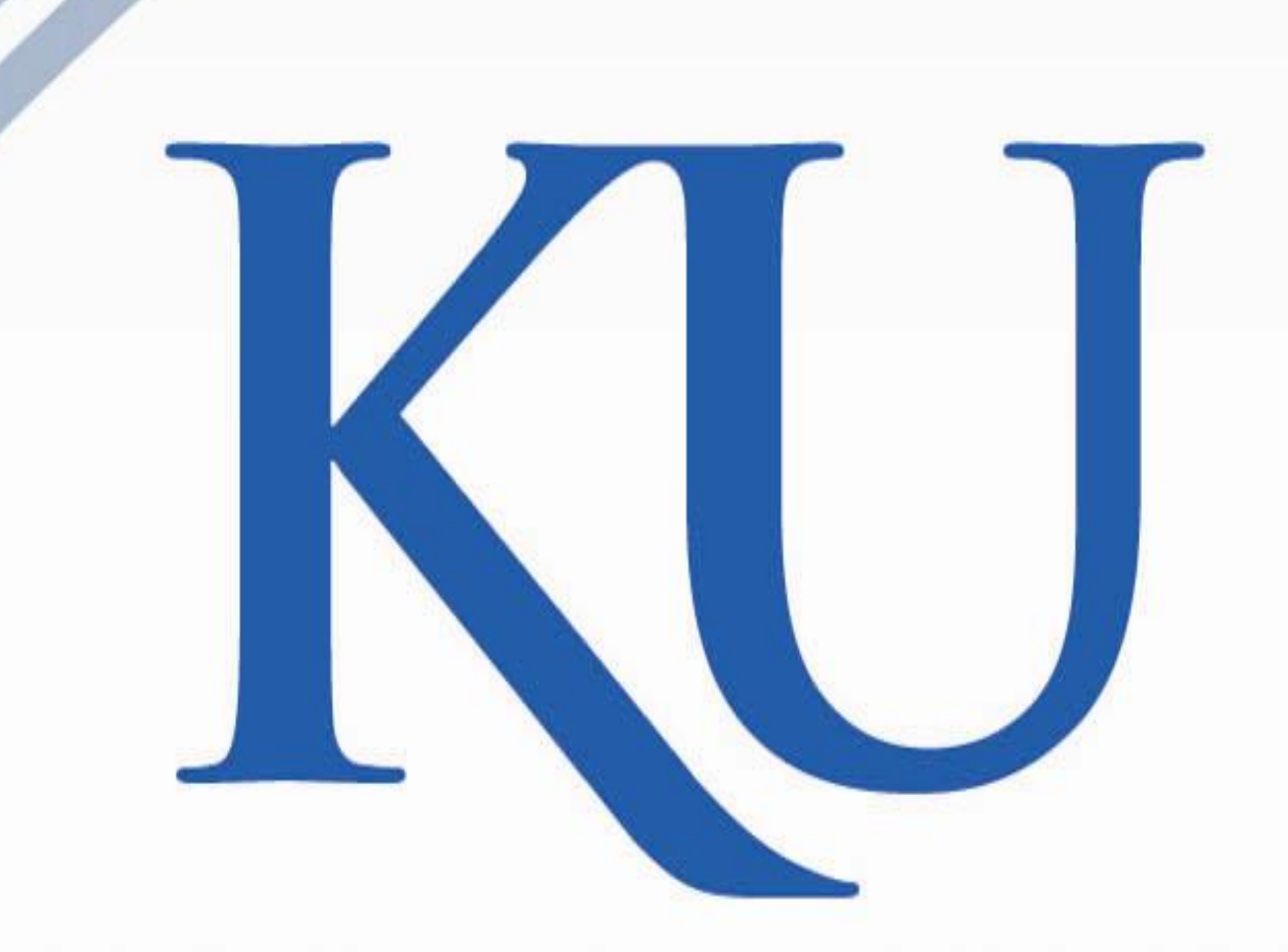

SCHOOL OF MEDICINE WICHITA

The University of Kansas 